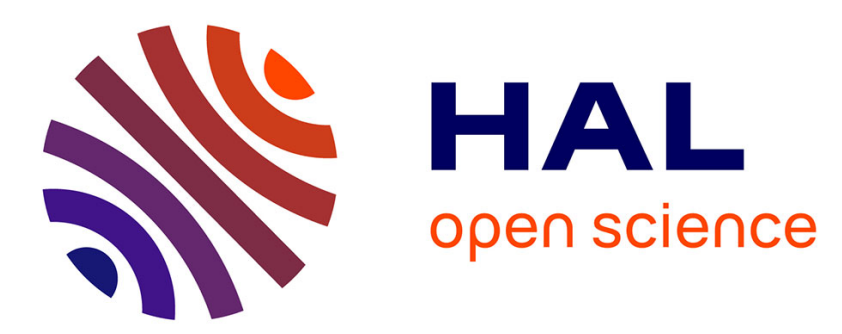

\title{
On the effect of lifetime variability on the performance of inventory systems
}

Chaaben Kouki, Oualid Jouini

\section{To cite this version:}

Chaaben Kouki, Oualid Jouini. On the effect of lifetime variability on the performance of inventory systems. International Journal of Production Economics, 2015, 167, pp.23-34. 10.1016/j.ijpe.2015.05.007 . hal-01265237

\section{HAL Id: hal-01265237 \\ https://hal.science/hal-01265237}

Submitted on 3 Feb 2016

HAL is a multi-disciplinary open access archive for the deposit and dissemination of scientific research documents, whether they are published or not. The documents may come from teaching and research institutions in France or abroad, or from public or private research centers.
L'archive ouverte pluridisciplinaire HAL, est destinée au dépôt et à la diffusion de documents scientifiques de niveau recherche, publiés ou non, émanant des établissements d'enseignement et de recherche français ou étrangers, des laboratoires publics ou privés. 


\title{
On the Effect of Lifetime Variability on the Performance of Inventory
}

\author{
Systems \\ Chaaben Kouki ${ }^{* 1}$ and Oualid Jouini ${ }^{2}$ \\ ${ }^{1}$ ESC Rennes School of Business, France, chaaben.kouki@gmail.fr \\ ${ }^{2}$ Laboratoire Génie Industriel, Ecole Centrale Paris, France, oualid.jouini@ecp.fr, text, \\ International Journal of Production Economics. To appear, 2015.
}

\begin{abstract}
We consider a periodic review perishable inventory system. Item lifetimes are random and assumed to follow an Erlang distribution. This is a rich modeling allowing to cover various cases of lifetime variability in practice. The demand process is assumed to be Poisson and the order lead time is constant. For the extreme cases of exponential and deterministic lifetimes, we provide an analytical approach for the system performance evaluation. We also conduct a simulation study to examine the important effect of lifetime variability mixed with the cost parameters on the total operating cost. It appeared that there might be opportunities to considerably improve the operating cost in the case of high lifetime variability. This is more apparent for cases with large outdate, lost sale, and purchasing cost parameters.
\end{abstract}

Keywords: Periodic review; Perishable items; Markov Process; Erlang lifetimes; inventory models.

${ }^{*}$ Corresponding author Tel: $+33(0) 299456817$; Fax : +33(0)299330824; e-mail: chaaben.kouki@gmail.fr 


\section{Introduction}

The operations management of perishable inventory systems has been mainly stimulated by the economic impact of perishability. In the grocery and pharmaceutical industry, expiration is responsible of $19 \%$ and $20 \%$ of total unsaleable respectively (Joint Industry Unsaleables Benchmark Survey, 2008). Lystad et al. (2006) reported that about $\$ 30$ billion are lost due to perishability in US grocery industry. Although the tradeoff between customer satisfaction and cost minimization could be handled through appropriate standard inventory control rules, the randomness of the item lifetimes makes such rules unsuccessful (Karaesmen et al., 2011). In this paper, we study the effect of the lifetime variability on the performance of inventory systems with perishable items. The application we have in mind is that of grocery products with no printed expiration date. Examples of such products include fresh fruits, vegetables, flowers and seafoods (Xiao and Chen, 2012). The change in the storage conditions (temperature, humidity, light exposure, etc.) considerably affect an item lifetime. This may create in turn a high variability in the items remaining lifetimes. We focus here on the study of the impact of such variability on the system performance, i.e., to what extent such variability may affect the inventory system operating costs? The answer to this question would help managers to assess the usefulness of investing on improving storage conditions in order to reduce the lifetime variability.

In this paper, we address the problem of perishable inventory management where the lifetime is modeled as a random variable. This allows to capture real-life situations of lifetime time variability. We consider the periodic review policy $(T, r, Q)$. The inventory level is periodically observed at the beginning of equal intervals of time with length $T$ each. If, and only if, at the observation epoch the inventory level is at or below the reorder point $r$, a replenishment order of $Q$ units is placed. As it is very often the case in practice, we choose a periodic review inventory scheme instead of a continuous one. For instance, hospitals typically order blood products from a central blood center periodically with regular order at the beginning of the ordering cycle and emergency order during the cycle (Zhou et al., 2011). Moreover, Material Requirement Planning and Automated Store Ordering System order a regular batch from suppliers at fixed intervals of time (Shang and Zhou, 2010; van Donselaar et al., 2010). Finally the $(T, r, Q)$ policy allows a cost reduction of the ordering and transportation costs by ordering items together with a common base period of review.

We consider Erlang distributions for lifetimes, which allows to cover a wide range of variability by varying the number of phases. The coefficient of variation may vary from the extreme value 1 to the 
extreme value 0 . The first extreme value corresponds to the case of an exponential distribution (high variability). This is reasonable for systems where item lifetimes are typically small but there are occasionally long lifetimes. The second extreme value corresponds to the case of a deterministic distribution (no variability), namely cases with perfectly respected storage and transportation conditions. The main contributions of this paper can be summarized as follows.

- For the extreme cases of exponential and deterministic lifetime distribution, we develop an analytical approach for the system performance evaluation during the steady-state regime. Our approach for the exponential case relies on the use of embedded Markov chains, and that for the deterministic case relies on the use of the regenerative property of the underlying process. For the two extreme cases, we compute the expected inventory level, the rates of orders, perished items and lost sales. Under an economic framework, this allows us next to optimize the total operating cost. To the best of our knowledge, the periodic review $(T, r, Q)$ policy for perishable inventory systems with stochastic lifetimes has not been studied yet. In Table 1, we provide a comparison between the key papers dealing with perishable inventory systems. The comparison is based on the features of the considered models. In this paper, we consider a periodic $(T, r, Q)$ replenishment policy, Poisson arrivals, lost sales, a deterministic lead time, a general lifetime distribution and an infinite planning horizon.

- For the general case with Erlang distributed lifetimes, we conduct a comprehensive simulation study that examines the important effect of the lifetime variability on the operating costs. It appeared that there might be opportunities for managers to considerably improve the operating cost in the case of high lifetime variability. This is more apparent for cases with large outdate, lost sale, and purchasing cost parameters.

The remainder of the paper is organized as follows. In Section 2, we review the literature of perishable inventory management related to this work. In Section 3, we describe the considered inventory model and concretely define the objective of the paper. In Section 4, we develop an analytical analysis for the particular cases of exponential and deterministic lifetimes. We also provide some numerical experiments to compare between them. In Section 5, we conduct a comprehensive analysis on the impact of lifetime variability using simulation. In Section 6, we give concluding remarks and highlight some future research. 


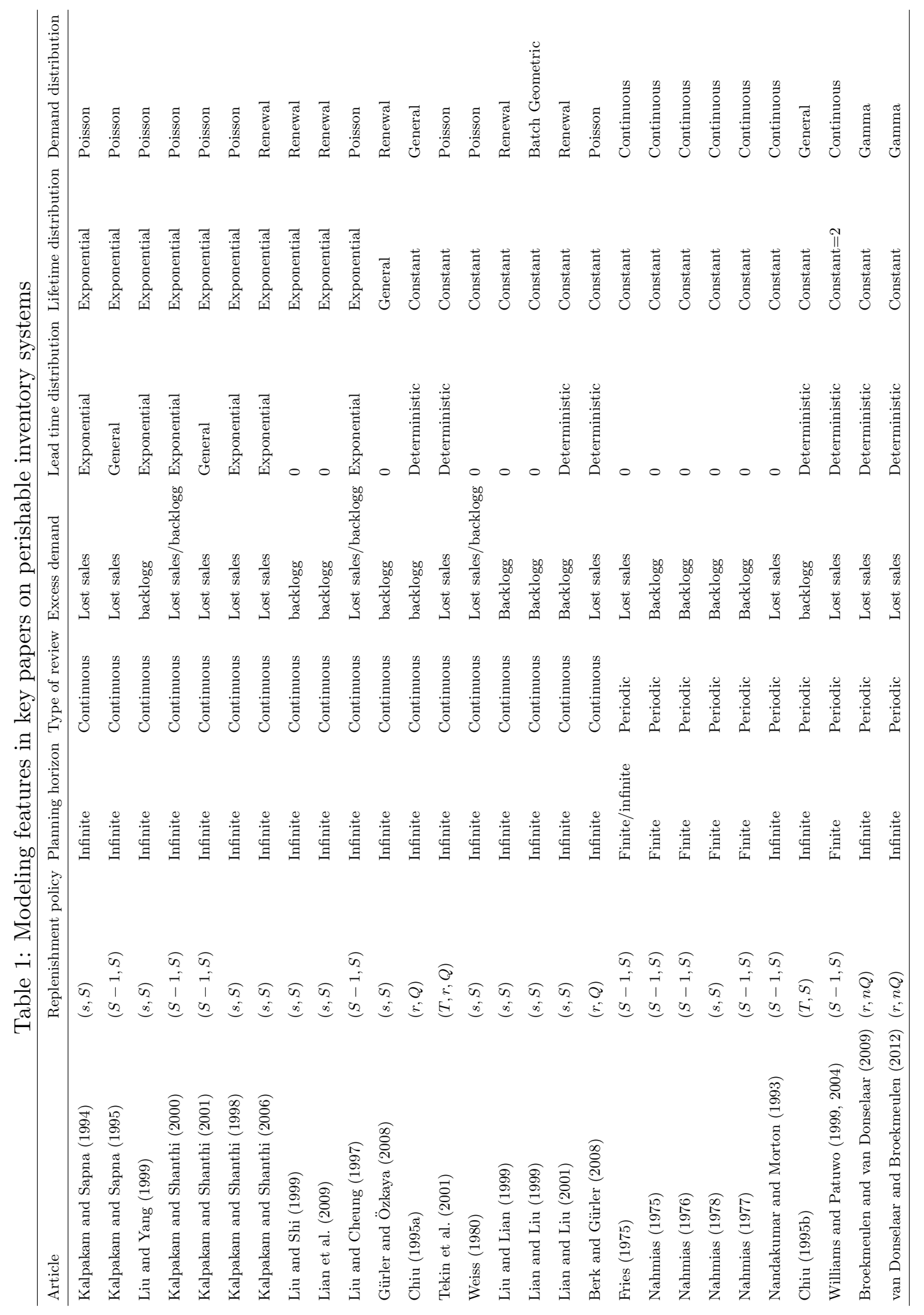




\section{Literature Review}

The analysis of inventory systems with perishable items has received significant attention to date in the literature. The effect of perishability on inventory management is studied either by combining inventory management with discounted pricing policies, either by an investment on technologies that help to preserve items on stock, or by seeking for the best inventory replenishment policies. Among the studies focusing on dynamic pricing for perishables inventory systems, one may find Herbon et al. (2014) and Chew et al. (2014), and Jia and $\mathrm{Hu}$ (2011). Works dealing with investment on technologies to track/evaluate the item lifetime include those by Piramuthu and Zhou (2013), Grunow and Piramuthu (2013), Wang and Li (2012), and Dye (2013). For investigations focusing on the best ordering policies, we refer the reader to Bakker et al. (2012), Karaesmen et al. (2011), and Nahmias (2011) for comprehensive surveys on periodic and continuous review policies for perishable inventory systems. When focusing on perishable inventory systems under stationary demand distribution, one can classify them into various classes depending on whether the product lifetime is deterministic or stochastic, the review policy is periodic or continuous, and the replenishment lead time is positive or instantaneous.

For deterministic lifetime models, most of works consider a periodic review and use the dynamic programming approach to find the optimal ordering quantity. Such analyses highlight the difficulty to track the different age's categories of items in stock since more variables are needed to track these remaining items and their ages to find the optimal order quantity Nahmias (2011). To overcome this complexity, approximate policies have been proposed in literature. For instance, Haijema et al. (2007, 2009) and Haijema (2013) use a combination of dynamic programming and simulation to reduce the state space and provide "near optimal" order-up-to-level inventory policies. The order-up-to-level policies are bounded by a maximum and a minimum and work only under the case of Poisson demand distribution. van Donselaar and Broekmeulen (2012) focus on the periodic review $(r, n Q)$ policy and propose an approximation of the outdating quantity by combining stochastic modeling, simulation and regression modeling.

Under a continuous review $(s, S)$ policy and assuming instantaneous replenishment lead times, Weiss (1980) followed by Lian and Liu (2001) and Lian et al. (2009) use the Markov renewal processes and the concept of regenerative processes in order to derive the optimal parameters. Heuristics to manage the case of positive lead times are proposed in Lian and Liu (2001), Chiu (1995b), Kouki et al. (2013) and in van Donselaar and Broekmeulen (2012). Tekin et al. (2001) introduce the age-based inventory 
policy which is suitable for particular items that start perishing when the order is unpacked for use. Duan and Liao (2013) proposed also an age-based policy to reduce waste of perishables. More recently, Berk and Gürler (2008) derive a closed form expression of the total ordering cost under the $(r, Q)$ inventory policy with lost sales and positive lead times. One of the analytical findings in this paper considers the case of deterministic lifetimes under the $(T, r, Q)$ policy with positive and constant lead times, for which no results exist yet.

Most of the work, in the literature dealing with random lifetimes, considers exponentially distributed lifetimes. Kalpakam and Sapna (1994) are the first to study an $(s, S)$ model for a Poisson demand distribution where product lifetimes and lead times are assumed to follow exponential distributions. Under these assumptions, the underlying inventory process is Markovian. By assuming lost sales and restricting the number of outstanding replenishment orders to, at most, one at any given time, they derive the steady-state probabilities and obtained the exact cost function and some useful analytical properties regarding the reorder point. Similar models are also investigated in Kalpakam and Shanthi (2000), Kalpakam and Shanthi (2001), Kalpakam and Shanthi (2006), Lian et al. (2009) and in Kouki et al. (2014). Another new analytical finding in this paper considers the case of exponential lifetimes under the $(T, r, Q)$ policy with positive and constant lead times.

There is an analogy between perishable inventory systems and queueing models with impatient customers. Known results from the queueing literature may be then used under certain conditions. For instance, Ioannidis et al. (2012) studied a make-to-stock production systems that operate under an $(S-1, S)$ policy for perishable item and impatient customers. The use of queuing models is initiated by Graves (1982) who analyzes perishable inventory systems where customer and orders arrive according to a Poisson processes. When all demand requests are for the same quantity and without considering any explicit ordering policy, Graves shows that the inventory process is equivalent to the virtual waiting time process for an $\mathrm{M} / \mathrm{D} / 1$ queue with customer abandonment. The similarity is easy to understand because the resupply time process can be seen as the server, the inventory as the queue, and the demand request as the customer arriving at the queue. If customers arrive according to a Poisson process and request an exponential batch size, the inventory process is equivalent to the virtual waiting time process for an $M / M / 1$ queue with customer abandonment. Finch (1960) shows that the sequence of waiting time of customers in the queue form a Markov chain with a finite state space. He derives the steady-state probability of the waiting time for any customer in the queuing system $G / M / 1+D$. Kaspi and Perry $(1983,1984)$ introduce the concept of virtual death-process based on the analysis of an M/G/1 queue with impatient customer. 
The virtual death-process is simply a reformulation of the age of the oldest item in stock used by Graves (1982). This concept is used by Perry and Stadje (1999) who derive explicit expressions of the stationary distribution of two models where arrival of items and demands are state-dependent and customers are willing to wait. This work is generalized in Nahmias et al. (2004) by deriving the steady-state distribution of the virtual outdating process in the context where the demand rate depends on the current value of the basic virtual outdating process.

\section{Modeling and Objective}

Consider a stochastic inventory system with a single supplier, which makes a single product (item) type to stock in order to satisfy a random demand. The arrival of customers follows a Poisson process with rate $\lambda$. Each arriving customer requests one unit of product. The replenishment (lead) time is assumed to be deterministic and is denoted by $L$.

Products cannot be infinitely stored. Each stored item has a random duration of time before it perishes. The time to perish is referred to as the lifetime. A perished item in the stock is unusable and is then discarded from the system as soon as it perishes. Product lifetimes are independent, identically distributed and follow an $m$-Erlang distribution with rate $\delta$, where $m$ is the number of phases, $m \geq 1$. Under the $m$-Erlang distribution, the time to perish of an item consists of $m$ phases, each phase is exponentially distributed with rate $m \delta$. The expected time to perish for an item is then $1 / \delta$. The family of $m$-Erlang distributions provides a modeling flexibility allowing therefore to cover various cases in practice. The exponential distribution is a special case of the $m$-Erlang distribution when $m=1$. The exponential distribution is common in modeling randomness in inventory operations. It is reasonable for systems with high lifetime variability where lifetimes are typically small but there are occasionally long lifetimes. As $m$ increases, the $m$-Erlang becomes more symmetrical, and as $m$ approaches $\infty$, lifetime becomes deterministic. The $m$-Erlang distribution also allows us to model lifetimes with a wide range of variability. In particular, by increasing $m$ while keeping $\delta$ constant, we can reduce the coefficient of variation of lifetimes (equal to $1 / \sqrt{m}$ ) while keeping the mean lifetime (equal to $1 / \delta$ ) constant.

Upon arrival, a customer is immediately satisfied by one available stored item, if any. If not, the customer is lost, i.e., leaves the system without service (loss of goodwill). The stored items are sold under the first in, first out (FIFO) discipline of service. This means that items made to stock earlier are sold first. The motivation of the FIFO rule assumption comes from a common practice in supermarkets, as 
mentioned in Tekin et al. (2001). Often, only one age category is exposed on the shelves to customers, and the remaining inventory is kept in a separate storage area. Recall also that we consider in this paper item examples with no printed expiration dates. For such items (vegetables, seafoods, etc.), it is often not easy for customers to identify the age category or the level of freshness. Therefore, even if items from different orders are mixed on the shelves, it suffices for the supermarket to put the items on the shelves such that the more recently arrived items are the less accessible ones to customers. This makes FIFO an appropriate assumption. Finally note that if there is complete information about residual lifetimes, the FIFO rule may not be an optimal policy (the shortest remaining lifetime first should behave better). However such information on residual lifetimes, of the items carried in stock, would require the use of advanced technology allowing to continuously update the remaining lifetime as a function of the evolution of some environment parameters (Piramuthu and Zhou, 2013; Grunow and Piramuthu, 2013). Such a technology is often expensive and then not available in most cases in practice. With no updated information on remaining lifetimes, FIFO is an appropriate policy. It enables to remove in expectation the oldest stored items, which allows to keep in stock the items with longer expected remaining lifetimes.

The system employs a periodic $(T, r, Q)$ policy to control the inventory level. It works as follows. The inventory level is periodically observed at the beginning of equal intervals of time with length $T$ each. If, and only if, at the observation epoch the inventory level is at or below the reorder point $r$, a replenishment order of $Q$ units is placed. The inventory level is then ranging from 0 to $r+Q$. For tractability issues, we assume in this paper that the period length $T$ is such that $L \leq T$. This ensures that, at most, one order is outstanding at any point of time. In using this assumption, we are following longstand practice in the modeling of perishable inventory models such as in Tekin et al. (2001) and in Berk and Gürler (2008). Without loss of generality, we assume that the inventory level is initially $Q$. An illustration of the inventory under the $(T, r, Q)$ policy is provided in Figure 1.

We build an economic framework as follows. The system incurs a fixed ordering cost $K$ per order, a purchasing cost $C$ per item unit, a holding cost $H$ per time unit for each stored item, an outdate cost $W$ per each perished item, and a lost sale cost $B$ for each customer arriving at an empty stock (this cost parameter reflects a loss of goodwill).

We next formulate a cost minimization problem. For $0 \leq i \leq r+Q$, let us denote by $P(i)$ the long run probability that the inventory level is $i$ just after an eventual order arrival, i.e., at the time points $\{n T+L, n=0,1,2 \ldots\}$. We denote by $p_{i, j}(t)$ the transition probability from state (inventory level) $i$ to state $j$ during an interval of time with length $t$, for $0 \leq i, j \leq r+Q$. Averaging over all possibilities, the 


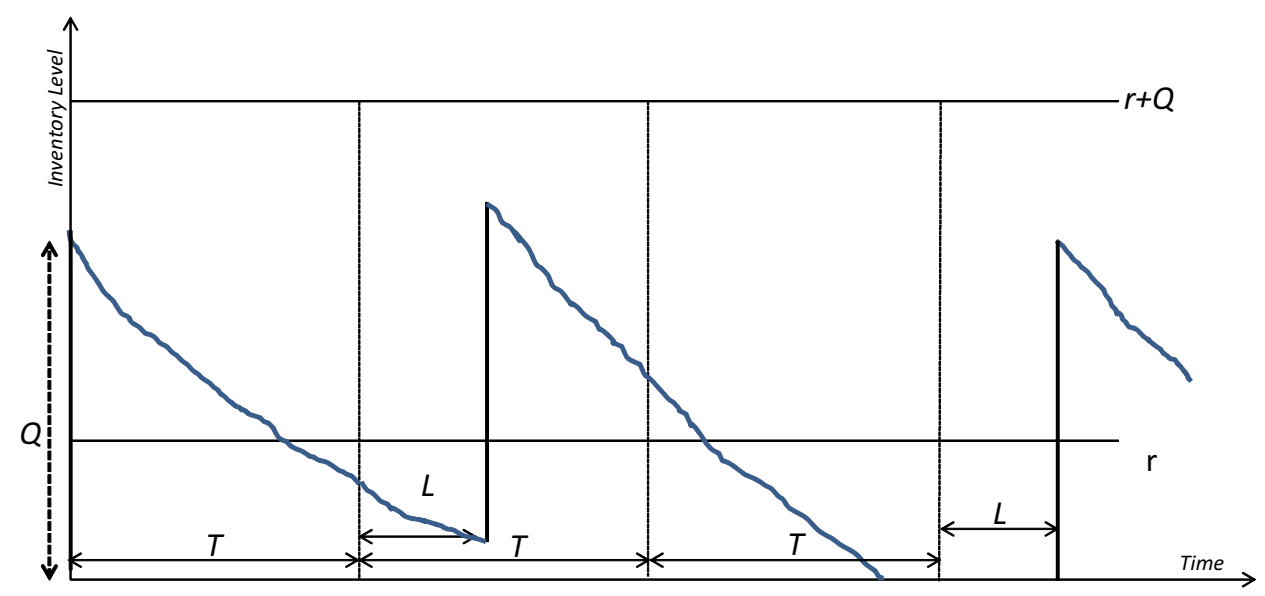

Figure 1: The $(T, r, Q)$ periodic review inventory policy

probability that an order is trigged at an arbitrary review point of time (beginning of an arbitrary period with length $T$ ) may be then written as $\sum_{i=0}^{r+Q} \sum_{j=0}^{r} P(i) \cdot p_{i, j}(T-L)$. We also define $E(I), E(O)$ and $E(S)$ as the long run expected inventory level, the long run expected rate of outdating items and the long run expected rate of lost sales, respectively. Hence, the total long run cost rate of the system, denoted by $T C(T, r, Q)$, is given by

$$
T C(T, r, Q)=\frac{K+C \cdot Q}{T} \sum_{i=0}^{r+Q} \sum_{j=0}^{r} P(i) \cdot p_{i, j}(T-L)+H \cdot E(I)+W \cdot E(O)+B \cdot E(S) .
$$

The objective is to find the optimal values of the policy parameters $T, r$ and $Q$, which minimize the steady-state total cost rate $T C(T, r, Q)$. We also want to examine the effect of the variability of the lifetime distribution on this optimal cost. For ease of referencing, Table 2 provides a summary of the notations used throughout the paper.

\section{Analytical Analysis of Particular Cases}

The analytical analysis of the model described in Section 3 is too complex. We consider in this section two particular cases of exponential (high variability) and deterministic (zero variability) lifetime distributions, for which, we develop exact results. The objective of this analysis is to obtain some sense on the effect of the lifetime variability on the total optimal cost. This allows also to obtain lower and upper bounds of the total optimal cost for the general case. A more comprehensive analysis is then conducted in Section 5 using simulation. 
Table 2: List of Notations

\begin{tabular}{ll}
\hline$\lambda:$ & Mean demand rate \\
$1 / \delta:$ & Mean lifetime of products \\
$m:$ & Number of phases for the Erlang distributed lifetimes \\
$L:$ & Replenishment lead time (constant) \\
$K:$ & Fixed ordering cost per order \\
$H:$ & Holding cost per unit of product held in stock per unit of time \\
$C:$ & Purchase cost per unit of product \\
$B:$ & Lost sales cost per unit of product \\
$W:$ & Outdate cost per unit of product that perishes in stock \\
$E(O):$ & Expected rate of outdating items \\
$E(S):$ & Expected rate of lost sales \\
$E(I):$ & Expected inventory level per unit of time \\
$T^{*}, r^{*}, Q^{*}$ & Optimal review period, optimal reorder level and optimal order quantity, respectively \\
$T C^{*}$ & Optimal total cost rate of the system \\
$p_{i, j}(t):$ & Transition probability from state (inventory level) $i$ to state $j$ during an interval of time with length $t$ \\
$P(i)$ & Steady state probability that the inventory level is $i$ just after an eventual order arrival
\end{tabular}

\subsection{The Case of Exponential Lifetimes}

We consider the case of exponentially distributed lifetimes and develop an exact method to compute the steady-state total cost rate of the system. The method is based on the analysis of an embedded continuous-time Markov chain seen on the epoch when an order may arrive, from which we derive the steady-state probabilities of the inventory level. This allows to derive all the cost components thereafter.

Let us define the stochastic process, denoted by $I(t)$, of the on-hand inventory level for the specific intervals of time $n T+L \leq t<(n+1) T+L$. Recall that a replenishment may only occur at the epochs $\{n T+L, n=0,1,2 \ldots\}$. During an interval $n T+L \leq t<(n+1) T+L$, the events that may occur are either customer arrivals or product deteriorations. Since inter-arrival times and lifetimes are exponential, $I(t)$ is a continuous-time Markov chain. More specifically, it is a generalized pure death-process. Let us define the transition probability $p_{i, j}(t)=P\{I(t)=j \mid I(n T+L)=i, n T+L \leq t<(n+1) T+L\}$, for $0 \leq i, j \leq r+Q$ and $n \geq 0$. Writing the Kolmogorov's Forward Equations implies

$$
\frac{d p_{i, j}(t)}{d t}= \begin{cases}-(\lambda+i \delta) p_{i, j}(t) & \text { if } i=j \\ -(\lambda+j \delta) p_{i, j}(t)+(\lambda+(j+1) \delta) p_{i, j+1}(t) & \text { if } 0 \leq j \leq i-1 \\ 0 & \text { otherwise }\end{cases}
$$

for $0 \leq i, j \leq r+Q$ and $n T+L \leq t<(n+1) T+L$. We denote by $\widetilde{p}_{i, j}(z)$, the Laplace transform of 
$p_{i, j}(t)$, for $z \in \mathbb{R}^{+}$. Applying the Laplace transform to Equations (2) leads to

$$
\begin{cases}(z+\lambda+i \delta) p_{i, j}(z)=0 & \text { if } i=j \\ (z+\lambda+j \delta) p_{i, j}(z)=(\lambda+(j+1) \delta) p_{i, j+1}(z) & \text { if } 0 \leq j \leq i-1\end{cases}
$$

for $0 \leq i, j \leq r+Q$ and $z \in \mathbb{R}^{+}$. Taking the initial condition $p_{i, i}(0)=1$, for $0 \leq i \leq r+Q$, and recursively solving Equations (3), we obtain

$$
\widetilde{p}_{i, j}(z)=\frac{1}{\lambda+j \delta} \prod_{k=j}^{i} \frac{\lambda+k \delta}{z+\lambda+k \delta}
$$

for $0 \leq i, j \leq r+Q$ and $z \in \mathbb{R}^{+}$. This equation has $i-j$ simple poles at $-(\lambda+k \delta), k=j, j+1, \ldots, i$. The residue at these poles can be expressed as

$$
\left.(z+\lambda+k \delta) \widetilde{p}_{i, j}(z)\right|_{z=-(\lambda+k \delta)}=e^{-(\lambda+k \delta) t} \prod_{n=j}^{k-1} \frac{1}{(n-k) \delta} \prod_{n=k+1}^{i} \frac{1}{(n-k) \delta}
$$

Using Equation (5) and after some algebra, the inversion of Equation (4) implies

$$
p_{i, j}(t)= \begin{cases}\frac{e^{-(\lambda+i \delta) t}\left(e^{\delta t}-1\right)^{i-j} \prod_{k=j+1}^{i}(\lambda+k \delta)}{(i-j) ! \delta^{i-j}} & \text { if } 0<j \leq i-1, \\ 1-\sum_{k=1}^{i} p_{i, k}(t) & \text { if } j=0, \\ 0 & \text { otherwise, }\end{cases}
$$

for $0 \leq i, j \leq r+Q$ and $n T+L \leq t<(n+1) T+L$. This characterizes the transition probability from state $i$ at time $n T+L$ to state $j$ at time $t<(n+1) T+L$. Let us now compute the transition probability $p_{i, j}(T)$ from state $i$ at time $n T+L$ to state $j$ at time $(n+1) T+L$. The difficulty here is that a replenishment may occur at time $(n+1) T+L$. We define $a_{i, j}(L)$ as the transition probability from state $i$ at time $(n+1) T$ to state $j$ at time $(n+1) T+L$ just after a replenishment (if an order is triggered at time $(n+1) T)$. Starting from state $i$ at time $n T+L$, the probability transition from $i$ to $j$ at time $(n+1) T$ is given by $p_{i, j}(T-L)$. Now, at time $(n+1) T+L$ the replenishment of size $Q$ occurs if the state of the inventory position is less or equal to $r$ at time $(n+1) T$. Using Equation $(6), a_{i, j}(L)$ may be 
written as

$$
a_{i, j}(L)= \begin{cases}p_{i, j-Q}(L) & \text { if } 0 \leq i \leq r, Q \leq j \leq r+Q, j-i \leq Q \\ p_{i, j}(L) & \text { if } r+1 \leq i \leq r+Q, 0 \leq j \leq i \\ 0 & \text { otherwise }\end{cases}
$$

We are now ready to compute $p_{i, j}(T)$. Averaging over all possibilities, it is given by

$$
p_{i, j}(T)=\sum_{k=0}^{i} p_{i, k}(T-L) a_{k, j}(L),
$$

for $0 \leq i, j \leq r+Q$.

Steady-State Probability of Inventory Levels. Consider the discrete-time stochastic process $\left\{I_{n}=\right.$ $I(n T+L), n \geq 0\}$ which describes the inventory level on-hand at the specific epochs $n T+L$ for $n \geq 0$. It is easy to see that it is a discrete-time Markov chain. Moreover, $\left\{I_{n}, n \geq 0\right\}$ is finite, aperiodic and irreducible. Then its steady-state probabilities, $P(i)=\lim _{n \rightarrow \infty} \operatorname{Pr}\left\{I_{n}=i\right\}$, exist and are unique, for $0 \leq i \leq r+Q$. They are the solutions of the fixed-point equation $P=P \cdot U \cdot V$ and the normalization equation, where $P=\{P(i), 0 \leq i \leq r+Q\}$ is the vector of the equilibrium probabilities, and $U$ and $V$ are transition matrixes of order $(r+Q+1)$. They are given by

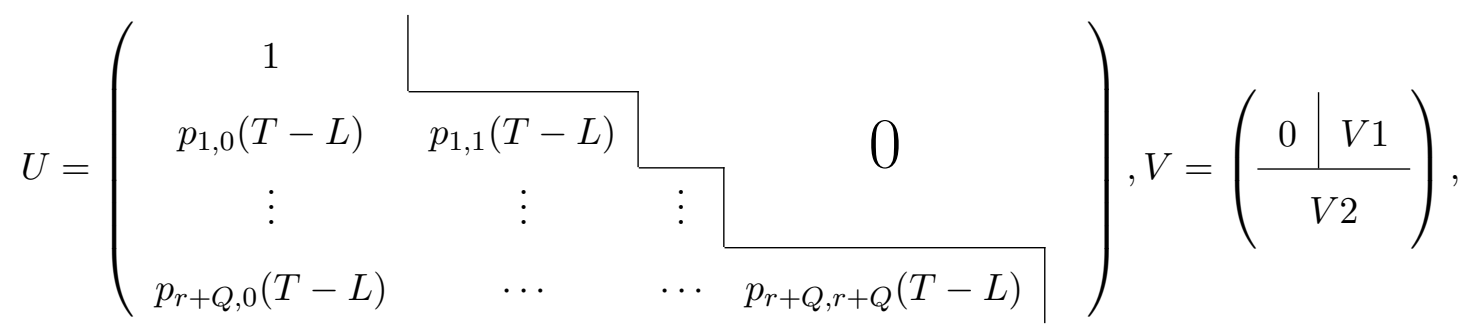

where $V 1$ and $V 2$ are the transition matrixes of size $(r+1) \times(r+1)$ and $Q \times(r+Q+1)$, respectively. They can be written as

$$
V 1=\left(\begin{array}{c|ccc}
1 & \multicolumn{3}{|c}{} \\
p_{1, Q}(L) & p_{1, Q+1}(L) & & \multicolumn{1}{c}{0} \\
\vdots & \vdots & \vdots & \\
p_{r, Q}(L) & \cdots & \cdots & p_{r, r+Q}(L)
\end{array}\right)
$$




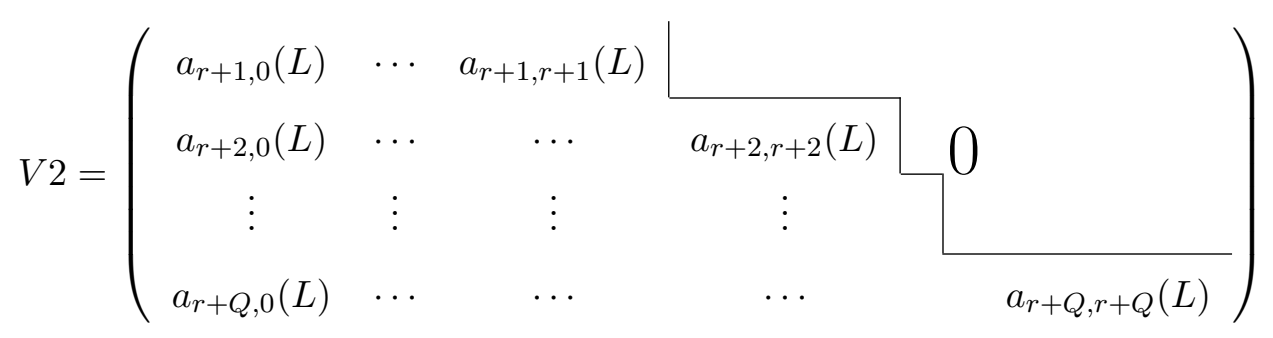

Thanks to the finite space, the numerical solving of the equation $P=P \cdot U \cdot V$ is straightforward. This finishes the characterization of $P(i)$ for $0 \leq i \leq r+Q$.

Expected Operating Costs. Consider the time interval with length $T$ that starts at time $n T+L$ (just after a replenishment, if any) and ends at $(n+1) T+L$ (just before a replenishment, if any), for $n \geq 0$. The inventory level at time $n T+L$ is $i$ with probability $P(i)$, for $0 \leq i \leq r+Q$. At any time $t, n T+L \leq t<(n+1) T+L$, it drops down to the level $j, 0 \leq j \leq i$, with probability $p_{i, j}(t)$, because of customer arrivals or product deteriorations. The conditional inventory level at that time $t$ is then $j$, given that the inventory level is $i$ at time $n T+L$. Averaging over all possibilities for state $i$, the expected inventory level per unit of time is thus given by

$$
E(I)=\frac{1}{T} \sum_{i=0}^{r+Q} P(i)\left(\sum_{j=0}^{i} j \int_{0}^{T} p_{i, j}(t) d t\right)
$$

Using the Markovian property of lifetimes, it follows that the expected rate of perished items is simply the expected inventory level times the lifetime rate. Hence

$$
E(O)=\delta E(I)
$$

Next, we compute the expected rate of lost sales. Following Hadley and Whitin (1963), we first compute the length of time per cycle $T$ (between time points $n T+L$ and $(n+1) T+L)$ during which the system is out of stock. We denote by $T_{S}$ this time of stockout, $0 \leq T_{S} \leq T$. The system starts with an inventory level $i$ at the beginning of the cycle, $i \geq 0$. We distinguish two cases. The first case is for $i=0$, for which we obviously have a stockout duration equal to the cycle duration, i.e., $T$. The second case is for $i \geq 1$. In this case, if the system reaches an out of stock condition in the time interval $t$ to $t+d t$, it means that in the time 0 to $t, i-1$ items have been either perished or asked by customers and the $i^{\text {th }}$ item is perished or asked by a customer between $t$ and $t+d t$. This occurs with probability $p_{i, 1}(t) \cdot(\lambda+\delta) d t$. If the system does reach an out of stock position between $t$ and $t+d t$, it will be out of stock for a duration $T-t$ during 
the cycle $T$. The above arguments lead to

$$
T_{S}=P(0) \cdot T+\sum_{i=1}^{r+Q} P(i)\left(\int_{0}^{T}(\lambda+\delta) \cdot(T-t) \cdot p_{i, 1}(t) d t\right) .
$$

The expected number of lost sales during $T$ is then the expected arrival rate times $T_{S}$. Therefore

$$
E(S)=\frac{\lambda T_{S}}{T}
$$

which implies

$$
E(S)=\lambda P(0)+\frac{\lambda}{T} \sum_{i=1}^{r+Q} P(i)\left(\int_{0}^{T}(\lambda+\delta) \cdot(T-t) \cdot p_{i, 1}(t) d t\right)
$$

Coming back to Equation (1), we obtain

$$
\begin{gathered}
T C(T, r, Q)=\frac{K+C Q}{T} \sum_{i=0}^{r+Q} \sum_{j=0}^{r} P(i) \cdot p_{i, j}(T-L)+\frac{H+\delta W}{T} \sum_{i=0}^{r+Q} \sum_{j=0}^{i} \int_{0}^{T} P(i) \cdot j \cdot p_{i, j}(t) d t \\
+\lambda \cdot B \cdot P(0)+\frac{\lambda B}{T} \cdot \sum_{i=1}^{r+Q} \int_{0}^{T} P(i) \cdot(\lambda+\delta) \cdot(T-t) \cdot p_{i, 1}(t) d t .
\end{gathered}
$$

Note that due to the memoryless property of lifetimes, the outdate cost $W$ in Equation (17) appears only in the element $H+\delta W$. For a given lifetime rate, this means that $W$ may be simply defined as a part of $H$. This completes the performance characterization under the case of exponentially distributed lifetimes.

\subsection{The Case of Deterministic Lifetimes}

We focus here on the case of deterministic lifetimes, i.e., we assume that an item perishes after exactly $\frac{1}{\delta}$ units of time. We distinguish three different cases: 1) $\left.\frac{1}{\delta}+L \leq T, 2\right) \frac{1}{\delta} \leq T<\frac{1}{\delta}+L$ and 3$) T<\frac{1}{\delta}$. For the first two conditions, we provide an exact method to compute the performance measures using the regenerative property of the inventory level at the replenishment epochs. The last case requires to keep track of the remaining lifetimes of items from different previous orders, which makes the analysis too complex.

Investigations focusing on how to keep track of the inventory on-hand for each lifetime level are described in Karaesmen et al. (2011) and in Nahmias (2011). For instance, Lian and Liu (2001) and Schmidt and Nahmias (1985) have demonstrated that a rigorous analysis would not be possible due to 
the excessive high dimensionality of the problem. This explains the emergence of several papers that take simplified assumptions such as a zero lead time, or approximations by for example ignoring the age of the "old" inventory at decision epochs. Such references include Chiu (1995a,b) who ignore the age distribution of the stored items. They only consider the information of the total inventory level at a decision epoch to derive approximate policies. Lian and Liu (2001) assume a zero lead time which allows to characterize their $(s, S)$ policy parameters. They next suggested a heuristic to deals with positive lead time. Further references for similar treatments can be found in Liu and Lian (1999), Gürler and Özkaya (2008), van Donselaar and Broekmeulen (2012), and Haijema (2013). In order to keep an exact analysis, we analytically analyze in this section the cases where the order $Q$ is totally exhausted before the arrival of a new order, i.e., for $T \geq \frac{1}{\delta}$. This ensures that a single age category of items at decision epochs. To overcome the difficulty of the analytical analysis of the case $T<\frac{1}{\delta}$, we resort to simulation in Section 5 .

Following the renewal reward theorem in Ross (1970), the expected rate of the total operating costs could be formulated as the expected cycle cost over the expected cycle length. In what follows, we compute this quantity for the two cases $\frac{1}{\delta}+L \leq T$ and $\frac{1}{\delta} \leq T<\frac{1}{\delta}+L$.

The case $\frac{1}{\delta}+L \leq T$. In this case, it is easy to see that the inventory level at the order arrival epoch will always start with a fresh batch $Q$. The regenerative cycle is therefore equal to $T$. After its arrival to the stock and prior to the time duration $\frac{1}{\delta}$, a removed item is necessarily asked by a customer (it has not reached its lifetime threshold). Recall that the arrival process is Poisson with rate $\lambda$. We then have

$$
E(I)=\sum_{j=0}^{Q} j \int_{0}^{\frac{1}{\delta}} p_{Q, j}(t) d t=\sum_{j=0}^{Q} j \int_{0}^{\frac{1}{\delta}} \frac{(\lambda t)^{Q-j} e^{-\lambda t}}{(Q-j) !} d t=\sum_{j=0}^{Q}(Q-j) \int_{0}^{\frac{1}{\delta}} \frac{(\lambda t)^{j} e^{-\lambda t}}{j !} d t
$$

Knowing that $\lambda \int_{0}^{t} \frac{(\lambda t)^{j} e^{-\lambda t}}{j !} d t=1-\sum_{i=0}^{j} \frac{(\lambda t)^{i} e^{-\lambda t}}{i !}$, for any positive real $t$ and any integer $j$, Equation (18) becomes

$$
\begin{aligned}
E(I) & =\sum_{j=0}^{Q}(Q-j) \frac{1}{\lambda}\left(1-\sum_{i=0}^{j} \frac{(\lambda / \delta)^{i} e^{-\lambda / \delta}}{i !}\right) \\
& =\frac{Q(Q+1)}{2 \lambda}-\frac{1}{\lambda} \sum_{j=0}^{Q} \sum_{i=0}^{j}(Q-j) \frac{(\lambda / \delta)^{i} e^{-\lambda / \delta}}{i !}
\end{aligned}
$$


Similarly, we can write

$$
E(O)=\sum_{j=0}^{Q}(Q-j) \cdot p_{Q, j}\left(\frac{1}{\delta}\right)=\sum_{j=0}^{Q}(Q-j) \frac{(\lambda / \delta)^{Q-j} e^{-\lambda / \delta}}{(Q-j) !}=\sum_{j=0}^{Q} \frac{(\lambda / \delta)^{j} e^{-\lambda / \delta}}{(j-1) !} .
$$

Also

$$
E(S)=\lambda\left(T-\frac{1}{\delta}\right)+\lambda\left(\int_{0}^{\frac{1}{\delta}} \lambda\left(\frac{1}{\delta}-t\right) \cdot p_{Q, 1}(t) d t\right)=\lambda\left(T-\frac{1}{\delta}\right)+\lambda\left(\int_{0}^{\frac{1}{\delta}} \lambda\left(\frac{1}{\delta}-t\right) \frac{e^{-\lambda t}(\lambda t)^{Q-1}}{(Q-1) !} d t\right)
$$

or equivalently after some algebra,

$$
E(S)=\lambda T-Q-\left(\frac{\lambda}{\delta}-Q\right) \sum_{j=0}^{Q-1} \frac{(\lambda / \delta)^{j} e^{-\lambda / \delta}}{j !}+\frac{(\lambda / \delta)^{Q} e^{-\lambda / \delta}}{(Q-1) !}
$$

Finally, the total operating cost is given by

$$
T C(T, r, Q)=\frac{K+C \cdot Q+H \cdot E(I)+W \cdot E(O)+B \cdot E(S)}{T} .
$$

The Case $\frac{1}{\delta} \leq T<\frac{1}{\delta}+L$. The main difference with the previous case is that here the regenerative cycle length depends on whether an order is triggered or not $T-L$ units of time after an order arrival. Then, the cycle length is $T$ with the probability that at least $Q-r$ items are removed from the stock, i.e., with probability $1-\sum_{j=0}^{Q-r-1} \frac{(\lambda(T-L))^{j} e^{-\lambda(T-L)}}{j !}$, and it is $2 T$ with the complementary probability. Let $E($ Cycle Length $)$ denotes the expected length of the regenerative cycle. It is then given by

$$
\begin{aligned}
E(\text { Cycle Length }) & =T\left(1-\sum_{j=0}^{Q-r-1} \frac{(\lambda(T-L))^{j} e^{-\lambda(T-L)}}{j !}\right)+2 T\left(\sum_{j=0}^{Q-r-1} \frac{(\lambda(T-L))^{j} e^{-\lambda(T-L)}}{j !}\right) \\
& =T\left(1+\sum_{j=0}^{Q-r-1} \frac{(\lambda(T-L))^{j} e^{-\lambda(T-L)}}{j !}\right) .
\end{aligned}
$$

Similarly to the previous case, the expected inventory level per time unit and the expected rate of perished items are given by Equations (19) and (20), respectively. The expected rate of lost sales is however different because it depends on the cycle length. By conditioning on the cycle length duration, it may be written 


$$
\begin{aligned}
E(S)= & \lambda\left(1-\sum_{j=0}^{Q-r-1} \frac{(\lambda(T-L))^{j} e^{-\lambda(T-L)}}{j !}\right)\left(T-\frac{1}{\delta}+\int_{0}^{\frac{1}{\delta}} \lambda\left(\frac{1}{\delta}-t\right) p_{Q, 1}(t) d t\right) \\
& +\lambda\left(\sum_{j=0}^{Q-r-1} \frac{(\lambda(T-L))^{j} e^{-\lambda(T-L)}}{j !}\right)\left(2 T-\frac{1}{\delta}+\int_{0}^{\frac{1}{\delta}} \lambda\left(\frac{1}{\delta}-t\right) p_{Q, 1}(t) d t\right) \\
= & \lambda T\left(1+\sum_{j=0}^{Q-r-1} \frac{(\lambda(T-L))^{j} e^{-\lambda(T-L)}}{j !}\right)-Q-\left(\frac{\lambda}{\delta}-Q\right) \sum_{j=0}^{Q-1} \frac{(\lambda / \delta)^{j} e^{-\lambda / \delta}}{j !}+\frac{(\lambda / \delta)^{Q} e^{-\lambda / \delta}}{(Q-1) !} .
\end{aligned}
$$

To obtain $T C(T, r, Q)$, it suffices to reuse Equation (23) by substituting the denominator by E(Cycle Length) given in Equation (24). This finishes the characterization of the total cost for the two cases $\frac{1}{\delta}+L \leq T$ and $\frac{1}{\delta} \leq T<\frac{1}{\delta}+L$

\subsection{Comparison}

We illustrate the usefulness of the developed analytical results by describing numerical experiments that compare between the two cases of deterministic and exponential lifetimes. The cost parameters setting we consider is: $K=\{10,100,150\}$ ( $€$ per order), $C=\{5,15\}$ (€ per item), $W=\{5,15\}$ (€ per perished item), $B=\{20,40\}$ (€ per lost customer) and $H=1$ (€ per stored item per time unit). The constant lead time is $L=1$ (time unit) and the expected arrival rate of customers is $\lambda=10$ (per time unit).

We assume that the expected lifetime is $\frac{1}{\delta}=3$ (time units). The cost parameters setting is similar to that in Chiu (1995b) and Berk and Gürler (2008). For each set of parameters, we compute the optimal review period, the optimal reorder point, the optimal order quantity and the optimal total operating cost, denoted by $T^{*}$ (time units), $r^{*}, Q^{*}$, and $T C^{*}$ (€ per time unit), respectively. Note that in all the numerical experiments, we consider values for $T^{*}$ that satisfy the condition $T^{*} \geq \frac{1}{\delta}$.

Because of the complicated expressions of the steady state probabilities, it is unfortunately very hard to derive structural results for the total cost function under the two cases of deterministic and exponential lifetimes. Such results would be helpful for the computation of the optimal parameters $T^{*}$, $r^{*}$ and $Q^{*}$. From the numerical experiments, we observe in general that the total cost function is not unimodal and it may have more than one local extremum. This is consistent with the observations made in Springael and Nieuwenhuyse (2005) and Hill and Johansen (2006). We then resort to an exhaustive search algorithm over a broad range of $(T, r, Q)$ values to numerically compute the optimal parameters. The values of $r$ and $Q$ are discrete, $0 \leq r \leq Q$ and $Q \geq 1$. To alleviate the computational efforts, we 
Table 3: Comparison between optimal costs for deterministic and exponential lifetimes $(\lambda=10,1 / \delta=3$, $L=1, H=1)$

\begin{tabular}{cccc|cccc|cccc|c}
\hline \multicolumn{1}{c|}{ Cost parameters } & \multicolumn{3}{|c|}{ Deterministic lifetime } & \multicolumn{3}{|c|}{ Exponential lifetime } & \multirow{2}{*}{$\frac{T C^{*}(E x p)-T C^{*}(\text { Det })}{T C^{*}(\text { Det })}$} \\
\hline$K$ & $C$ & $B$ & $W$ & $T^{*}$ & $r^{*}$ & $Q^{*}$ & $T C^{*}$ & $T^{*}$ & $r^{*}$ & $Q^{*}$ & $T C^{*}$ & $39 \%$ \\
\hline \hline 10 & 5 & 20 & 5 & 3 & 29 & 30 & 86.72 & 3 & 39 & 33 & 141.29 & $35 \%$ \\
50 & 5 & 20 & 5 & 3 & 29 & 30 & 100.05 & 3 & 27 & 33 & 154.63 & $32 \%$ \\
100 & 5 & 20 & 5 & 3 & 24 & 30 & 116.05 & 3 & 21 & 33 & 171.29 & $43 \%$ \\
10 & 5 & 40 & 5 & 3 & 31 & 33 & 96.88 & 3 & 49 & 48 & 169.42 & $40 \%$ \\
50 & 5 & 40 & 5 & 3 & 31 & 33 & 110.21 & 3 & 45 & 48 & 182.75 & $36 \%$ \\
100 & 5 & 40 & 5 & 3 & 31 & 33 & 126.88 & 3 & 42 & 48 & 199.42 & $44 \%$ \\
10 & 5 & 20 & 15 & 3 & 25 & 28 & 91.89 & 3 & 20 & 19 & 165.00 & $41 \%$ \\
50 & 5 & 20 & 15 & 3 & 25 & 28 & 105.22 & 3 & 18 & 19 & 178.33 & $37 \%$ \\
100 & 5 & 20 & 15 & 3 & 25 & 28 & 121.89 & 3 & 11 & 19 & 195.00 & $53 \%$ \\
10 & 5 & 40 & 15 & 3 & 29 & 31 & 107.84 & 3 & 40 & 39 & 227.34 & $50 \%$ \\
50 & 5 & 40 & 15 & 3 & 29 & 31 & 121.17 & 3 & 36 & 39 & 240.68 & $46 \%$ \\
100 & 5 & 40 & 15 & 3 & 29 & 31 & 137.84 & 3 & 33 & 39 & 257.34 & $12 \%$ \\
10 & 15 & 20 & 5 & 3 & 19 & 23 & 176.01 & 3 & 6 & 6 & 199.05 & $10 \%$ \\
50 & 15 & 20 & 5 & 3 & 19 & 23 & 189.35 & 4 & 0 & 6 & 209.29 & $9 \%$ \\
100 & 15 & 20 & 5 & 3 & 0 & 25 & 202.47 & 4 & 0 & 6 & 221.79 & $34 \%$ \\
10 & 15 & 40 & 5 & 3 & 29 & 30 & 201.24 & 3 & 33 & 31 & 304.65 & $33 \%$ \\
50 & 15 & 40 & 5 & 3 & 29 & 30 & 214.58 & 3 & 29 & 31 & 317.98 & $31 \%$ \\
100 & 15 & 40 & 5 & 3 & 29 & 30 & 231.24 & 3 & 25 & 31 & 334.65 & $12 \%$ \\
10 & 15 & 20 & 15 & 3 & 20 & 22 & 176.71 & 4 & 0 & 4 & 200.38 & $10 \%$ \\
50 & 15 & 20 & 15 & 3 & 20 & 22 & 190.05 & 4 & 0 & 4 & 210.38 & $9 \%$ \\
100 & 15 & 20 & 15 & 3 & 0 & 23 & 203.22 & 4 & 0 & 4 & 222.88 & $37 \%$ \\
10 & 15 & 40 & 15 & 3 & 28 & 29 & 207.19 & 3 & 23 & 22 & 330.36 & $36 \%$ \\
50 & 15 & 40 & 15 & 3 & 28 & 29 & 220.53 & 3 & 23 & 22 & 343.70 & $34 \%$ \\
100 & 15 & 40 & 15 & 3 & 28 & 29 & 237.19 & 3 & 20 & 22 & 360.36 & \\
\hline
\end{tabular}

restrict the optimization problem to discrete values of $T$ in steps of 1 . From all the obtained combinations for $(T, r, Q)$, we finally choose the one leading to the best total cost, $T C^{*}$. The results are given in Table 3.

Table 3 reveals that the total cost with deterministic lifetimes is always lower than that with exponential lifetimes. This agrees with the well known property that performance deteriorates with variability. For some combinations of the cost parameters the relative cost difference could be around $50 \%$. This implies for managers that it is important to take the appropriate actions allowing to decrease the variability in lifetimes, by for example improving or standardizing the storage conditions of items.

We also observe from Table 3 that some cases yield to $r^{*}=0$ when the lifetime is exponentially distributed. This means replenishing only when the inventory is depleted. The observation agrees with the result in Theorem 3 of Weiss (1980); because of the cost parameter values, it may be better to loose demand rather than to incur inventory holding and outdating costs. This observation suggests that managers should give a particular care in the estimation step of cost components. A wrong estimation would indeed lead to a poor inventory policy. 
For our general model with Erlang lifetimes, we conjecture that the extreme cases of deterministic and exponential lifetimes are lower and upper bounds of the total cost, respectively. A rigorous proof of this result is too complex, but numerical illustrations using simulation are given in the next section.

The cost upper and lower bounds are useful for the system manager to get some sense on her actual cost. However, one would like to go beyond these two extreme points in order to deeper examine the effect of lifetime variability. This is unfortunately not possible using an analytical analysis. We then conduct simulation experiments in the next section to investigate to what extent lifetime variability deteriorates performance.

\section{Simulation Experiments and Insights}

In this section, we conduct a simulation study for the general case as described in Section 3, i.e., lifetimes follow an $m$-Erlang distribution with mean $m / \delta$ and $m$ phases. When $m=1$, the lifetime is exponential. Since the coefficient of variation of the $m$-Erlang distribution is $1 / \sqrt{m}$, the lifetime distribution converges to the deterministic distribution with mean $1 / \delta$ as $m \longrightarrow \infty$. We therefore examine the impact of the lifetime randomness on the total cost, by varying the number of phases $m$.

We consider various sets in order to mix the effect of lifetime variability with the cost parameters. The cost parameters setting is the same as that used in Section 4.3. We control the variability of lifetimes by varying the number of phases $m$ from 1 (exponential distribution) to 10000 (nearly deterministic distribution). We use simulation-based optimization to compute the optimal policy parameters $T^{*}, r^{*}$ and $Q^{*}$. Simulation experiments run on Arena software, with the following sequence of events 1) An order arrives, 2) perished products are discarded, 3) demand is observed, 4) inventory Position is reviewed, 5) an order is triggered.

Similarly to Section 4.3, we use an exhaustive search algorithm over a broad range of $(T, r, Q)$ values to numerically compute the optimal parameters. The only difference here is that at each iteration, we use simulation to compute the total cost instead of using a numerical computation. The values of $r$ and $Q$ are discrete, $0 \leq r \leq Q$ and $Q \geq 1$. To alleviate the computational efforts, we again restrict the optimization problem to discrete values of $T$ in steps of 1 . For a given set of parameters, we run a single simulation for a sufficiently large duration denoted by $D$. The duration $D$ is chosen such that the performance measures do not vary for larger simulation durations, with a sufficiently high precision (four digits beyond the decimal point). For the considered settings, this is ensured with $D=20000$ units of 
time. The performance measures are computed as follows. The expected inventory level per time unit, $E(I)$, is computed as a time-persistent variable given by $E(I)=\frac{1}{D} \sum_{i=0}^{r+Q} i \cdot \Gamma(i, D)$, where $\Gamma(i, D)$ is the total time for which the inventory level is $i$. The expected rate of outdating items, $E(O)$ is computed as the total number of outdating items divided by the simulation duration $D$. Similarly, the expected rate of lost sales, $E(S)$, is computed as the total number of lost sales divided by $D$. Finally, the expected cycle length, E(Cycle Length), is computed as the expected value of all the realizations of the time durations between two successive orders. The results are given in Tables 4 and 5, and in Figures 2-7.

A general conclusion from the numerical results is that the lifetime variability have an important effect on the total cost. The experiments illustrate the conjecture that the total cost of the extreme deterministic and exponential lifetime cases are lower and upper bounds of the total cost of the Erlang lifetime cases, respectively. When the coefficient of variation decreases from 1 to 0.44 , the results indicate that the cost improvement ranges from $11 \%$ (for $K=50, C=15, B=20$ and $W=5$ ) to $46 \%$ (for $K=100, C=5, B=40$ and $W=15$ ). Thus, there might be opportunities for managers to considerably improve the operating cost by controlling the lifetime variability.

We observe that reducing lifetime variability has a diminishing return. Figures 2-5 reveal that most of the improvement in the total optimal cost takes place when moving from cases with high variability (coefficient of variation close to one). In other words, the lower is the variability, the lower is the sensitivity of the optimal cost. An important insight here is that an investment on reducing the item lifetime variability, through for instance the improvement of storage conditions, would be justified only for items with already non-negligible variability (larger than 0.1).

We also observe that the effect magnitude of the outdate cost $W$ depends on the lifetime variability. Figures 2-5 show that increasing the outdating cost significantly increases the total cost for only the cases with high lifetime variability. An explanation is as follows. To the contrary to the cases with low variability, the expected amount of perished products is important in the cases with high variability, and so is the corresponding cost component. Similarly, the performance deterioration with lifetime variability makes the total cost more sensitive to the lost sale cost parameter $B$ in the case of high variability than in those with low variability. Moreover, Figures 6 and 7 reveal that this sensitivity is further apparent in the case with high purchasing cost parameter $C$.

Finally, recall that the experiments shown so far are restricted to the case of a lead time that is lower than or equal to the period length, $L \leq T$. Simulation experiments without this restriction are provided in Figures 8 and 9. The experiments show that the conclusions found above still hold. 
Table 4: Optimal inventory policy $(\lambda=10,1 / \delta=3, L=1, H=1)$

\begin{tabular}{|c|c|c|c|c|c|c|c|c|c|c|c|c|c|c|c|c|c|c|c|}
\hline \multicolumn{4}{|c|}{ Cost Parameters } & \multicolumn{8}{|c|}{ Lifetime distribution, $m=1$} & \multicolumn{8}{|c|}{ Lifetime distribution, $m=50$} \\
\hline$K$ & $C$ & $B$ & $W$ & $T^{*}$ & $r^{*}$ & $Q^{*}$ & $E(I)$ & $E(O)$ & $E(S)$ & E(Cycle Length $)$ & $T C^{*}$ & $T^{*}$ & $r^{*}$ & $Q^{*}$ & $E(I)$ & $E(O)$ & $E(S)$ & E(Cycle Length $)$ & $T C^{*}$ \\
\hline 10 & 5 & 20 & 5 & 1 & 22 & 11 & 6.36 & 2.12 & 1.13 & 1.00 & 104.51 & 1 & 21 & 20 & 13.70 & 0.30 & 0.42 & 2.03 & 77.94 \\
\hline 50 & 5 & 20 & 5 & 1 & 17 & 24 & 10.24 & 3.41 & 1.47 & 2.01 & 141.28 & 1 & 18 & 22 & 14.14 & 0.39 & 0.43 & 2.22 & 96.86 \\
\hline 100 & 5 & 20 & 5 & 1 & 14 & 25 & 10.47 & 3.49 & 1.52 & 2.09 & 165.98 & 1 & 16 & 24 & 13.89 & 0.44 & 0.66 & 2.46 & 118.83 \\
\hline 10 & 5 & 40 & 5 & 1 & 26 & 13 & 9.86 & 3.29 & 0.37 & 1.01 & 115.56 & 1 & 22 & 21 & 15.69 & 0.51 & 0.26 & 2.05 & 84.50 \\
\hline 50 & 5 & 40 & 5 & 1 & 24 & 13 & 9.61 & 3.20 & 0.41 & 1.02 & 155.19 & 1 & 20 & 22 & 15.26 & 0.50 & 0.29 & 2.16 & 103.30 \\
\hline 100 & 5 & 40 & 5 & 1 & 22 & 29 & 14.75 & 4.92 & 0.56 & 2.02 & 183.16 & 1 & 19 & 24 & 15.94 & 0.63 & 0.31 & 2.33 & 125.96 \\
\hline 10 & 5 & 20 & 15 & 1 & 19 & 10 & 5.07 & 1.69 & 1.70 & 1.00 & 124.32 & 1 & 18 & 20 & 12.39 & 0.21 & 0.59 & 2.08 & 80.30 \\
\hline 50 & 5 & 20 & 15 & 1 & 15 & 10 & 4.94 & 1.65 & 1.80 & 1.02 & 164.10 & 1 & 16 & 20 & 11.59 & 0.17 & 0.74 & 2.13 & 99.47 \\
\hline 100 & 5 & 20 & 15 & 1 & 9 & 18 & 6.01 & 2.00 & 3.21 & 2.05 & 193.12 & 1 & 15 & 21 & 11.83 & 0.20 & 0.79 & 2.24 & 122.28 \\
\hline 10 & 5 & 40 & 15 & 1 & 24 & 12 & 7.95 & 2.65 & 0.68 & 1.00 & 144.82 & 1 & 21 & 21 & 15.07 & 0.45 & 0.29 & 2.07 & 89.07 \\
\hline 50 & 5 & 40 & 15 & 1 & 22 & 12 & 7.84 & 2.61 & 0.71 & 1.01 & 184.59 & 1 & 20 & 22 & 15.26 & 0.50 & 0.29 & 2.16 & 108.25 \\
\hline 100 & 5 & 40 & 15 & 1 & 19 & 26 & 11.91 & 3.97 & 1.05 & 2.01 & 227.62 & 1 & 20 & 22 & 15.26 & 0.50 & 0.29 & 2.16 & 131.38 \\
\hline 10 & 15 & 20 & 5 & 1 & 10 & 6 & 1.86 & 0.62 & 4.62 & 1.00 & 197.39 & 1 & 18 & 18 & 9.94 & 0.08 & 1.07 & 2.01 & 171.02 \\
\hline 50 & 15 & 20 & 5 & 4 & 0 & 6 & 0.46 & 0.15 & 8.65 & 4.00 & 209.29 & 1 & 11 & 20 & 9.15 & 0.08 & 1.71 & 2.40 & 189.63 \\
\hline 100 & 15 & 20 & 5 & 4 & 0 & 6 & 0.46 & 0.15 & 8.65 & 4.00 & 221.79 & 3 & 0 & 23 & 5.34 & 0.08 & 5.57 & 5.16 & 203.54 \\
\hline 10 & 15 & 40 & 5 & 1 & 24 & 11 & 6.37 & 2.12 & 1.13 & 1.00 & 237.01 & 1 & 21 & 20 & 13.70 & 0.30 & 0.42 & 2.03 & 185.10 \\
\hline 50 & 15 & 40 & 5 & 1 & 19 & 12 & 7.42 & 2.47 & 0.86 & 1.03 & 276.88 & 1 & 19 & 21 & 13.90 & 0.35 & 0.41 & 2.12 & 203.98 \\
\hline 100 & 15 & 40 & 5 & 1 & 16 & 24 & 10.18 & 3.39 & 1.49 & 2.02 & 314.96 & 1 & 18 & 22 & 14.14 & 0.39 & 0.43 & 2.22 & 227.31 \\
\hline 10 & 15 & 20 & 15 & 4 & 0 & 4 & 0.23 & 0.08 & 9.08 & 4.00 & 200.38 & 2 & 17 & 17 & 8.44 & 0.04 & 1.53 & 2.01 & 171.63 \\
\hline 50 & 15 & 20 & 15 & 4 & 0 & 4 & 0.23 & 0.08 & 9.08 & 4.00 & 210.38 & 1 & 10 & 20 & 8.63 & 0.07 & 2.03 & 2.50 & 190.34 \\
\hline 100 & 15 & 20 & 15 & 4 & 0 & 4 & 0.23 & 0.08 & 9.08 & 4.00 & 222.88 & 3 & 0 & 23 & 5.34 & 0.08 & 5.57 & 5.16 & 204.37 \\
\hline 10 & 15 & 40 & 15 & 1 & 19 & 11 & 6.27 & 2.09 & 1.17 & 1.01 & 258.13 & 1 & 19 & 20 & 12.90 & 0.25 & 0.53 & 2.06 & 188.03 \\
\hline 50 & 15 & 40 & 15 & 1 & 17 & 11 & 6.09 & 2.03 & 1.27 & 1.02 & 297.68 & 1 & 19 & 20 & 12.90 & 0.25 & 0.53 & 2.06 & 207.44 \\
\hline \multirow[t]{2}{*}{100} & 15 & 40 & 15 & 2 & 18 & 20 & 7.42 & 2.47 & 2.47 & 2.00 & 343.43 & 1 & 17 & 21 & 12.84 & 0.26 & 0.56 & 2.17 & 230.75 \\
\hline & & & & \multicolumn{8}{|c|}{ Lifetime distribution, $m=500$} & \multicolumn{8}{|c|}{ Lifetime distribution, $m=10000$} \\
\hline$K$ & $C$ & $B$ & $W$ & $T$ & $r$ & $Q$ & $E(I)$ & $E(O)$ & $E(S)$ & E(Cycle Length $)$ & $T C^{*}$ & $T$ & $r$ & $Q$ & $E(I)$ & $E(O)$ & $E(S)$ & E(Cycle Length $)$ & $T C^{*}$ \\
\hline 10 & 5 & 20 & 5 & 1 & 18 & 21 & 13.39 & 0.20 & 0.47 & 2.16 & 76.98 & 1 & 19 & 21 & 13.84 & 0.24 & 0.41 & 2.15 & 76.83 \\
\hline 50 & 5 & 20 & 5 & 1 & 18 & 22 & 14.16 & 0.29 & 0.39 & 2.22 & 95.30 & 1 & 18 & 22 & 14.19 & 0.28 & 0.39 & 2.23 & 95.08 \\
\hline 100 & 5 & 20 & 5 & 3 & 24 & 30 & 15.37 & 0.71 & 0.70 & 3.00 & 116.22 & 3 & 24 & 30 & 15.53 & 0.70 & 0.68 & 3.00 & 116.05 \\
\hline 10 & 5 & 40 & 5 & 1 & 21 & 22 & 15.84 & 0.45 & 0.24 & 2.16 & 83.41 & 1 & 21 & 22 & 15.68 & 0.43 & 0.24 & 2.16 & 83.05 \\
\hline 50 & 5 & 40 & 5 & 1 & 21 & 23 & 16.46 & 0.55 & 0.20 & 2.23 & 101.30 & 1 & 20 & 23 & 15.89 & 0.47 & 0.24 & 2.25 & 101.07 \\
\hline 100 & 5 & 40 & 5 & 1 & 21 & 24 & 17.19 & 0.66 & 0.17 & 2.29 & 123.26 & 1 & 20 & 24 & 16.63 & 0.60 & 0.20 & 2.31 & 122.81 \\
\hline 10 & 5 & 20 & 15 & 1 & 18 & 20 & 12.35 & 0.14 & 0.60 & 2.10 & 78.78 & 1 & 17 & 20 & 11.97 & 0.12 & 0.65 & 2.12 & 78.71 \\
\hline 50 & 5 & 20 & 15 & 1 & 16 & 21 & 12.41 & 0.15 & 0.63 & 2.21 & 97.39 & 1 & 17 & 21 & 12.87 & 0.17 & 0.54 & 2.18 & 97.18 \\
\hline 100 & 5 & 20 & 15 & 1 & 15 & 22 & 12.53 & 0.20 & 0.71 & 2.34 & 119.52 & 1 & 15 & 23 & 12.99 & 0.23 & 0.71 & 2.43 & 119.18 \\
\hline 10 & 5 & 40 & 15 & 1 & 19 & 22 & 14.60 & 0.33 & 0.34 & 2.21 & 87.43 & 1 & 20 & 22 & 15.13 & 0.36 & 0.29 & 2.19 & 87.02 \\
\hline 50 & 5 & 40 & 15 & 1 & 19 & 22 & 14.60 & 0.33 & 0.34 & 2.21 & 105.57 & 1 & 20 & 22 & 15.13 & 0.36 & 0.29 & 2.19 & 105.33 \\
\hline 100 & 5 & 40 & 15 & 1 & 18 & 23 & 14.80 & 0.39 & 0.34 & 2.29 & 128.16 & 1 & 19 & 23 & 15.36 & 0.42 & 0.28 & 2.27 & 127.44 \\
\hline 10 & 15 & 20 & 5 & 1 & 14 & 18 & 9.22 & 0.02 & 1.27 & 2.07 & 170.08 & 1 & 14 & 18 & 9.20 & 0.02 & 1.27 & 2.07 & 169.95 \\
\hline 50 & 15 & 20 & 5 & 1 & 11 & 20 & 9.18 & 0.03 & 1.67 & 2.40 & 188.38 & 1 & 11 & 23 & 10.29 & 0.10 & 1.73 & 2.77 & 188.18 \\
\hline 100 & 15 & 20 & 5 & 3 & 0 & 25 & 5.84 & 0.08 & 5.54 & 5.56 & 202.55 & 3 & 0 & 25 & 5.83 & 0.08 & 5.54 & 5.56 & 202.47 \\
\hline 10 & 15 & 40 & 5 & 1 & 20 & 21 & 14.25 & 0.28 & 0.38 & 2.13 & 183.44 & 1 & 19 & 21 & 13.84 & 0.24 & 0.41 & 2.15 & 182.92 \\
\hline 50 & 15 & 40 & 5 & 1 & 19 & 22 & 14.68 & 0.33 & 0.33 & 2.21 & 201.87 & 1 & 18 & 22 & 14.19 & 0.28 & 0.39 & 2.23 & 201.46 \\
\hline 100 & 15 & 40 & 5 & 1 & 18 & 22 & 14.16 & 0.29 & 0.39 & 2.22 & 224.47 & 1 & 18 & 22 & 14.19 & 0.28 & 0.39 & 2.23 & 223.88 \\
\hline 10 & 15 & 20 & 15 & 1 & 14 & 18 & 9.23 & 0.02 & 1.27 & 2.07 & 170.36 & 1 & 14 & 18 & 9.20 & 0.02 & 1.27 & 2.07 & 170.20 \\
\hline 50 & 15 & 20 & 15 & 1 & 10 & 20 & 8.63 & 0.03 & 2.03 & 2.52 & 188.87 & 1 & 11 & 20 & 9.19 & 0.03 & 1.68 & 2.41 & 188.66 \\
\hline 100 & 15 & 20 & 15 & 3 & 0 & 23 & 5.37 & 0.04 & 5.54 & 5.15 & 203.28 & 3 & 0 & 23 & 5.37 & 0.04 & 5.54 & 5.15 & 203.22 \\
\hline 10 & 15 & 40 & 15 & 1 & 18 & 21 & 13.39 & 0.20 & 0.47 & 2.16 & 185.47 & 1 & 18 & 21 & 13.42 & 0.21 & 0.46 & 2.16 & 185.25 \\
\hline 50 & 15 & 40 & 15 & 1 & 18 & 21 & 13.39 & 0.20 & 0.47 & 2.16 & 203.95 & 1 & 18 & 21 & 13.42 & 0.21 & 0.46 & 2.16 & 203.74 \\
\hline 100 & 15 & 40 & 15 & 1 & 18 & 21 & 13.30 & 0.21 & 0.47 & 2.17 & 227.02 & 1 & 17 & 22 & 13.65 & 0.24 & 0.47 & 2.26 & 226.71 \\
\hline
\end{tabular}




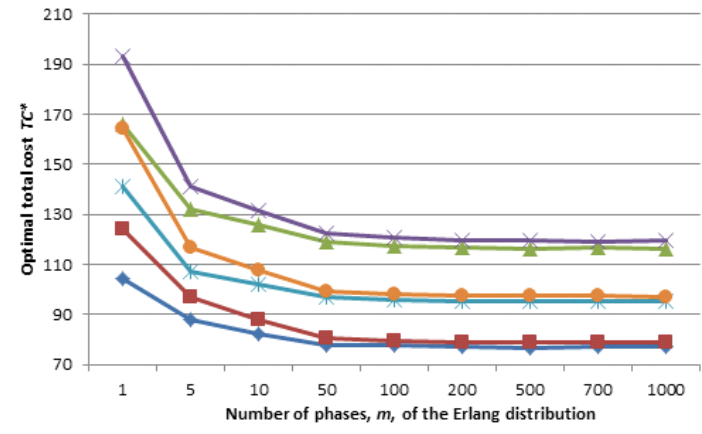

Figure 2: Optimal total cost for $C=5, B=20$ and $H=1(\lambda=10,1 / \delta=3, L=1)$

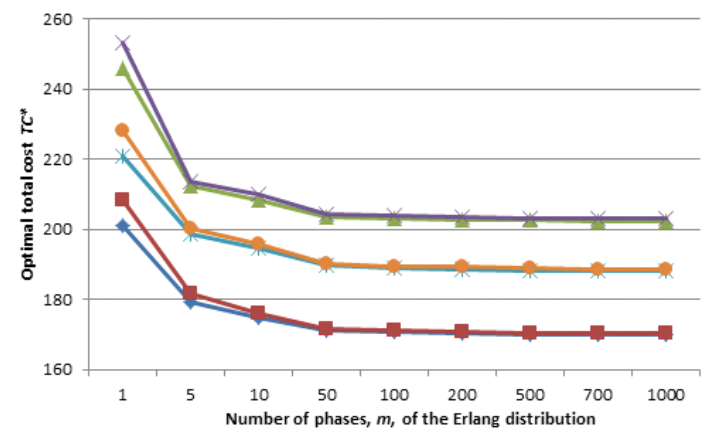

Figure 4: Optimal total cost for $C=15 B=20$ and $H=1(\lambda=10,1 / \delta=3, L=1)$

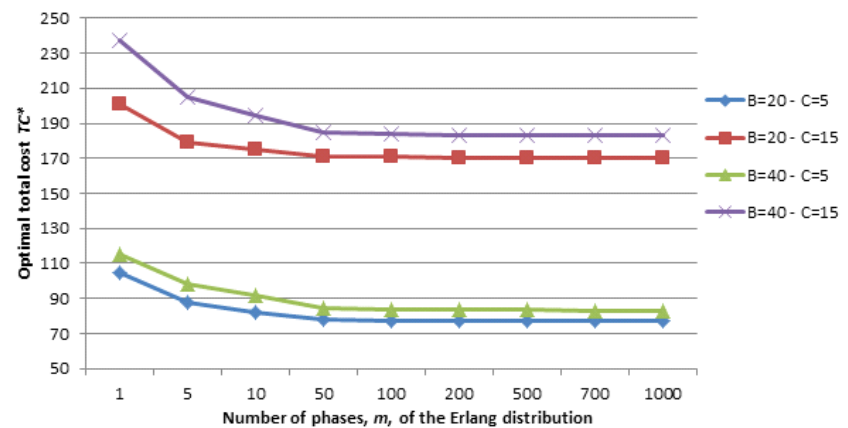

Figure 6: Optimal total cost for $W=5, K=10$ and $H=1(\lambda=10,1 / \delta=3, L=1)$

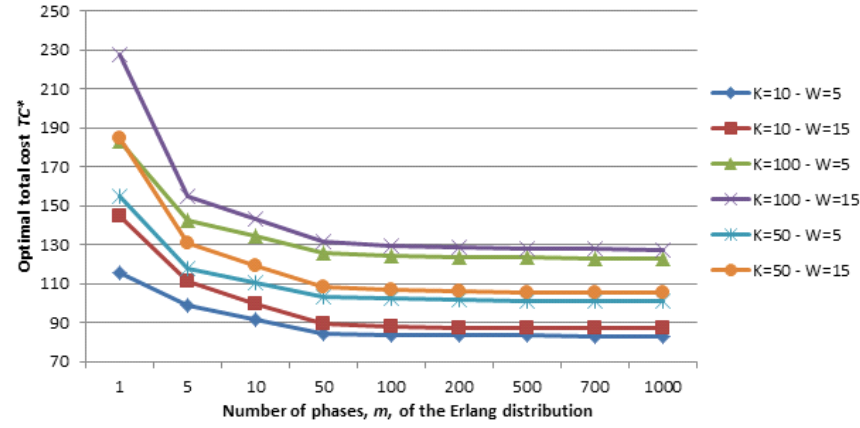

Figure 3: Optimal total cost for $C=5, B=40$ and $H=1(\lambda=10,1 / \delta=3, L=1)$

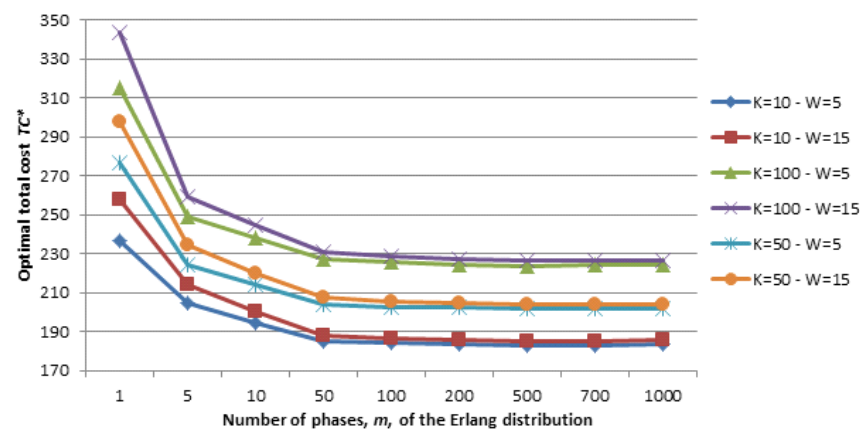

Figure 5: Optimal total cost for $C=15, B=40$ and $H=1(\lambda=10,1 / \delta=3, L=1)$

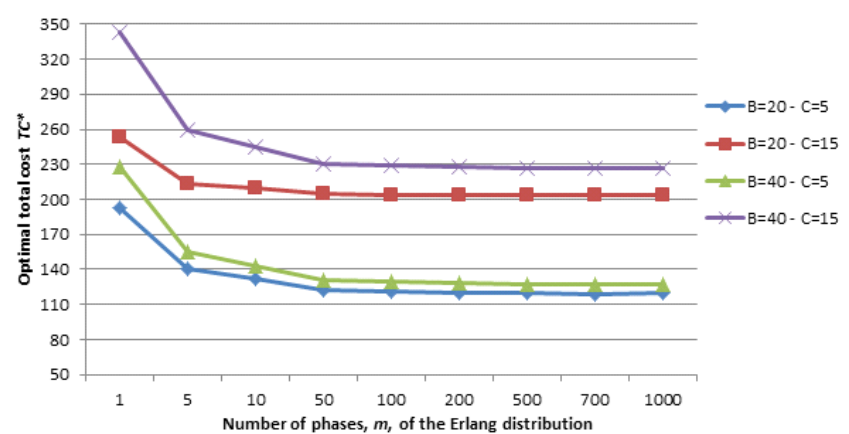

Figure 7: Optimal total cost for $W=15, K=100$ and $H=1(\lambda=10,1 / \delta=3, L=1)$ 


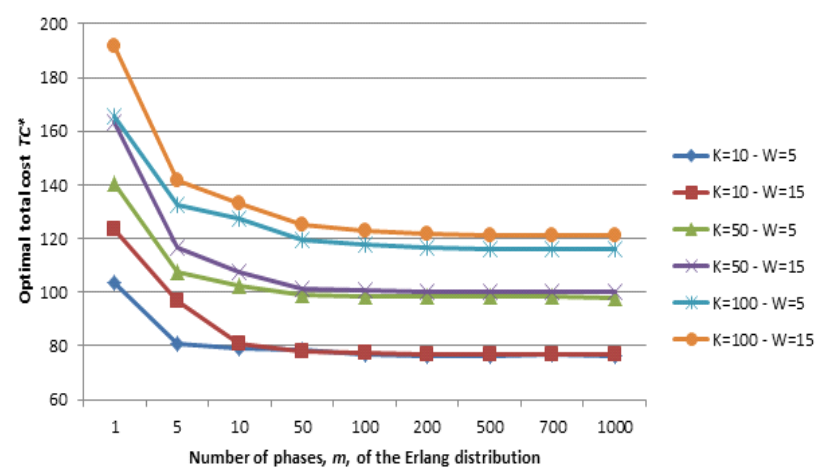

Figure 8: Optimal total cost for $C=5, B=20$ and Figure 9: Optimal total cost for $W=5, C=5$ and $H=1(\lambda=10,1 / \delta=3, L=3)$

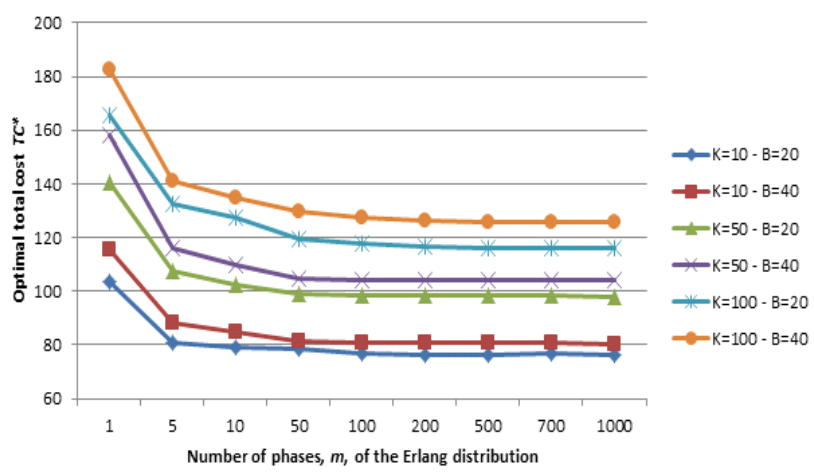
$H=1(\lambda=10,1 / \delta=3, L=3)$

Table 5: Effect of the number of phases, $m$, on the optimal total cost $(\lambda=10,1 / \delta=3, L=1, H=1)$

\begin{tabular}{|c|c|c|c|c|c|c|c|c|c|c|c|c|c|}
\hline & $C$ & \multicolumn{6}{|c|}{5} & \multicolumn{6}{|c|}{15} \\
\hline & $B$ & \multicolumn{3}{|c|}{20} & \multicolumn{3}{|c|}{40} & \multicolumn{3}{|c|}{20} & \multicolumn{3}{|c|}{40} \\
\hline & $K$ & 10 & 50 & 100 & 10 & 50 & 100 & 10 & 50 & 100 & 10 & 50 & 100 \\
\hline \multirow{10}{*}{$W=5$} & $m=1$ & 104.51 & 141.28 & 165.98 & 115.56 & 155.19 & 183.16 & 197.39 & 209.29 & 221.79 & 237.01 & 276.88 & 314.96 \\
\hline & $m=5$ & 87.63 & 107.34 & 131.89 & 98.50 & 118.06 & 142.26 & 179.45 & 198.82 & 212.27 & 204.74 & 224.45 & 249.10 \\
\hline & $m=10$ & 82.37 & 101.93 & 125.64 & 91.46 & 110.56 & 134.42 & 174.71 & 194.51 & 208.30 & 194.46 & 214.10 & 238.05 \\
\hline & $m=50$ & 77.94 & 96.86 & 118.83 & 84.50 & 103.30 & 125.96 & 171.02 & 189.63 & 203.54 & 185.10 & 203.98 & 227.31 \\
\hline & $m=100$ & 77.42 & 95.97 & 117.53 & 83.68 & 102.37 & 124.38 & 170.64 & 188.84 & 203.07 & 183.94 & 202.70 & 225.62 \\
\hline & $m=200$ & 77.11 & 95.44 & 116.64 & 83.57 & 101.88 & 123.74 & 170.28 & 188.73 & 202.68 & 183.52 & 202.13 & 224.57 \\
\hline & $m=500$ & 76.98 & 95.30 & 116.22 & 83.41 & 101.30 & 123.26 & 170.08 & 188.38 & 202.55 & 183.44 & 201.87 & 224.47 \\
\hline & $m=700$ & 76.91 & 95.24 & 116.14 & 83.33 & 101.22 & 123.03 & 170.04 & 188.35 & 202.51 & 183.28 & 201.83 & 224.27 \\
\hline & $m=1000$ & 76.86 & 95.20 & 116.09 & 83.09 & 101.16 & 122.87 & 170.03 & 188.25 & 202.48 & 183.03 & 201.55 & 224.15 \\
\hline & $m=10000$ & 76.83 & 95.08 & 116.05 & 83.05 & 101.07 & 122.81 & 169.95 & 188.18 & 202.47 & 182.92 & 201.46 & 223.88 \\
\hline \multirow{10}{*}{$W=15$} & $m=1$ & 124.32 & 164.10 & 193.12 & 144.82 & 184.59 & 227.62 & 200.38 & 210.38 & 222.88 & 258.13 & 297.68 & 343.43 \\
\hline & $m=5$ & 96.69 & 116.51 & 141.01 & 111.34 & 130.74 & 154.99 & 181.58 & 200.38 & 213.66 & 214.35 & 234.17 & 258.95 \\
\hline & $m=10$ & 87.67 & 107.47 & 131.67 & 99.33 & 118.91 & 143.39 & 176.05 & 196.01 & 210.08 & 200.20 & 219.99 & 244.69 \\
\hline & $m=50$ & 80.30 & 99.47 & 122.28 & 89.07 & 108.25 & 131.38 & 171.63 & 190.34 & 204.37 & 188.03 & 207.44 & 230.75 \\
\hline & $m=100$ & 79.40 & 98.16 & 120.77 & 88.13 & 106.69 & 129.69 & 171.10 & 189.47 & 203.75 & 186.80 & 205.35 & 228.54 \\
\hline & $m=200$ & 78.80 & 97.45 & 119.68 & 87.48 & 105.83 & 128.61 & 170.66 & 189.19 & 203.44 & 185.67 & 204.34 & 227.40 \\
\hline & $m=500$ & 78.78 & 97.39 & 119.52 & 87.43 & 105.57 & 128.16 & 170.36 & 188.87 & 203.28 & 185.47 & 203.95 & 227.02 \\
\hline & $m=700$ & 78.78 & 97.30 & 119.45 & 87.08 & 105.38 & 127.73 & 170.32 & 188.71 & 203.27 & 185.45 & 203.94 & 226.76 \\
\hline & $m=1000$ & 78.72 & 97.25 & 119.36 & 87.05 & 105.37 & 127.69 & 170.26 & 188.70 & 203.25 & 185.29 & 203.92 & 226.72 \\
\hline & $m=10000$ & 78.71 & 97.18 & 119.18 & 87.02 & 105.33 & 127.44 & 170.20 & 188.66 & 203.22 & 185.25 & 203.74 & 226.71 \\
\hline
\end{tabular}




\section{Conclusions and Future Research}

We considered a $(T, r, Q)$ lost sales inventory system with perishable items. Item lifetimes are random and assumed to follow an Erlang distribution. This is a rich modeling allowing to cover various cases of lifetime variability in practice. For the extreme cases of exponential and deterministic lifetimes, we provided an analytical approach for the system performance evaluation during the steady-state regime, mainly the expected inventory level, the rates of orders, perished items and lost sales. This allowed to obtain some sense on the effect of the lifetime variability on the total optimal cost. A more comprehensive analysis for the general case is then conducted using simulation. We have examined the important effect of lifetime variability mixed with the cost parameters on the total operating cost. It appeared that there might be opportunities for managers to considerably improve the operating cost in the case of high lifetime variability. This is more apparent for cases with large outdate, lost sale, and purchasing cost parameters.

In a future research, it would be useful to extend the analytical analysis to the Erlang distributed lifetimes. Another interesting work is to prove the stochastic ordering between systems with different lifetime variabilities. An ambitious future work would be to extend the model we developed herein to the case of one warehouse multiple retailers inventory systems for perishables. We believe that the work of Shang and Zhou (2010) could be extended to the case of perishables with random lifetime and insights regarding the impact of perishability on the optimal policy could be examined. 


\section{References}

Avinadav, T., Herbon, A., and Spiegel, U. (2013). Optimal inventory policy for a perishable item with demand function sensitive to price and time. International Journal of Production Economics, 144(2):497 $-506$.

Bakker, M., Riezebos, J., and Teunter, R. (2012). Review of inventory systems with deterioration since 2001. European Journal of Operational Research, 221(2):275 - 284.

Berk, E. and Gürler, Ü. (2008). Analysis of the (Q,r) inventory model for perishables with positive lead times and lost sales. Operations Research, 56:1238-1246.

Broekmeulen, R. A. C. M. and van Donselaar, K. H. (2009). A heuristic to manage perishable inventory with batch ordering, positive lead-times, and time-varying demand. Computers and Operations Research, 36:3013-3018.

Chew, E., Lee, C., Liu, R., Hong, K., and Zhang, A. (In press. 2014). Optimal dynamic pricing and ordering decisions for perishable products. International Journal of Production Economics.

Chiu, H. (1995a). An approximation to the continuous review inventory model with perishable items and lead times. European Journal of Operational Research, 87:93-108.

Chiu, H. (1995b). A heuristic (R, T) periodic review perishable inventory model with lead times. International Journal of Production Economics, 42:1-15.

Duan, Q. and Liao, T. W. (2013). A new age-based replenishment policy for supply chain inventory optimization of highly perishable products. International Journal of Production Economics, 145(2):658 $-671$.

Dye, C. (2013). The effect of preservation technology investment on a non-instantaneous deteriorating inventory model. Omega, 41(5):872 - 880 .

Finch, P. D. (1960). Deterministic customer impatience in the queueing system GI/M/1. Biometrika, $47: 45-52$.

Fries, B. (1975). Optimal order policy for a perishable commodity with fixed lifetime. Operations Research, $23: 46-61$. 
Graves, S. (1982). The application of queueing theory to continuous perishable inventory systems. Management Science, 28:400-406.

Grunow, M. and Piramuthu, S. (2013). RFID in highly perishable food supply chains remaining shelf life to supplant expiry date? International Journal of Production Economics, 146(2):717 - 727.

Gürler, Ü. and Özkaya, B. (2008). Analysis of the (s, S) policy for perishables with a random shelf life. IIE Transactions, 40:759-781.

Hadley, G. and Whitin, T. M. (1963). Analysis of Inventory Systems. Prentice-Hall, Englewood Cliffs, NJ, USA.

Haijema, R. (2013). A new class of stock-level dependent ordering policies for perishables with a short maximum shelf life. International Journal of Production Economics, 143(2):434 - 439.

Haijema, R., van der Wa, J., and van Dijk, N. (2007). Blood platelet production: Optimization by dynamic programming and simulation. Computers and Operations Research, 34(3):760 - 779 .

Haijema, R., van Dijk N.M., van der Wal J., and Sibinga, C. (2009). Blood platelet production with breaks: optimization by SDP and simulation. International Journal of Production Economics, 121(2):464 - 473.

Herbon, A., Levner, E., and Cheng, T. (2014). Perishable inventory management with dynamic pricing using timetemperature indicators linked to automatic detecting devices. International Journal of Production Economics, 147:605-613.

Hill, R. and Johansen, S. (2006). Optimal and near-optimal policies for lost sales inventory models with at most one replenishment order outstanding. European Journal of Operational Research, 169(1):111 132.

Ioannidis, S., Jouini, O., Economopoulos, A., and Kouikoglou, V. (2012). Control policies for single-stage production systems with perishable inventory and customer impatience. Annals of Operations Research, 209(1):115-138.

Jia, J. and Hu, Q. (2011). Dynamic ordering and pricing for a perishable goods supply chain. Computers and Industrial Engineering, 60(2):302 - 309.

Joint Industry Unsaleables Benchmark Survey (2008). The real causes and actionable solutions. Technical report. www.gmaonline.org/downloads/research-and-reports/UnsaleablesFINAL091108.pdf. 
Kalpakam, S. and Sapna, K. (1994). Continuous review (s, S) inventory system with random lifetimes and positive leadtimes. Operations Research Letters, 16:115-119.

Kalpakam, S. and Sapna, K. (1995). (S-1, S) perishable systems with stochastic lead times. Mathematical and Computer Modelling, 21:95-104.

Kalpakam, S. and Shanthi, S. (1998). A continuous review perishable system with positive lead time. International Journal of Management and Systems, 14:123-134.

Kalpakam, S. and Shanthi, S. (2000). Perishable system with modified base stock policy and random supply quantity. Computations in Mathematics with Applications, 39:79-89.

Kalpakam, S. and Shanthi, S. (2001). A perishable inventory system with modified (S-1, S) policy and arbitrary processing times. Computers and Operations Research, 28:453-471.

Kalpakam, S. and Shanthi, S. (2006). A continuous review perishable system with renewal demand. Annals of Operations Research, 143:211-225.

Karaesmen, I., Scheller-Wolf, A., and Deniz, B. (2011). Managing perishable and aging inventories: Review and future research directions. Planning Production and Inventories in the Extended Enterprise: A State of the Art Handbook, K. Kempf, P. Keskinocak, R. Uzsoy (Eds.) International Series in Operations Research and Management Science. Springer, 151:393-436.

Kaspi, H. and Perry, D. (1983). Inventory systems of perishable commodities. Advances in Applied Probability, 15:674-685.

Kaspi, H. and Perry, D. (1984). Inventory systems for perishable commodities with renewal input and poisson output. Advances in Applied Probability, 16:402-421.

Kouki, C., Sahin, E., Jema, Z., and Dallery, Y. (2013). Assessing the impact of perishability and the use of time temperature technologies on inventory management. International Journal of Production Economics, 143(1):72 - 85.

Kouki, C., Sahin, E., Jema, Z., and Dallery, Y. (2014). Analysis of a periodic review inventory control system with perishables having random lifetime. International Journal of Production Research, $52(1): 283-298$. 
Lian, Z. and Liu, L. (1999). A discrete-time model for perishable inventory systems. Annals of Operations Research, 87:103-116.

Lian, Z. and Liu, L. (2001). Continuous review perishable inventory systems: Models and heuristics. IIE Transactions, 33:809-822.

Lian, Z., Liu, X., and Zhao, N. (2009). A perishable inventory model with markovian renewal demands. International Journal of Production Economic, 121:176-182.

Liu, L. and Cheung, K. (1997). Service constrained inventory models with random lifetimes and lead times. The Journal of the Operational Research Society, 48:1022-1028.

Liu, L. and Lian, Z. (1999). (s, S) continuous review models for products with fixed lifetimes. Operations Research, 47:150-158.

Liu, L. and Shi, D. (1999). An (s, S) model for inventory with exponential lifetimes and renewal demands. Naval Research Logistics, 46:39-56.

Liu, L. and Yang, T. (1999). An (s, S) random lifetime inventory model with a positive lead time. European Journal of Operational Research, 113:52-63.

Lystad, E., Ferguson, M., and Alexopoulos, C. (2006). Single stage heuristic for perishable inventory control in two-echelon supply chains. Working paper. Georgia Institute of Technology. https://smartech.gatech.edu/handle/1853/11677?show=full.

Nahmias, S. (1975). Optimal ordering policies for perishable inventory. Operations Research, 23:735-749.

Nahmias, S. (1976). Myopic approximations for the perishable inventory problem. Management Science, 22:1002-1008.

Nahmias, S. (1977). Higher order approximations for the perishable inventory problem. Operations Research, 25:630-640.

Nahmias, S. (1978). The fixed charge perishable inventory problem. Operations Research, 26:464-481.

Nahmias, S. (2011). Perishable Inventory Systems. Springer.

Nahmias, S., Perry, D., and Stadje, W. (2004). Perishable inventory systems with variable input and demand rates. Mathematical Methods of Operations Research, 60:155-162. 
Nandakumar, P. and Morton, T. (1993). Near myopic heuristics for the fixed-life perishable problem. Management Science, 39:1490- 1498.

Perry, D. and Stadje, W. (1999). Perishable inventory systems with impatient demands. Mathematical Methods of Operations Research, 50:77-90.

Piramuthu, S. and Zhou, W. (2013). RFID and perishable inventory management with shelf-space and freshness dependent demand. International Journal of Production Economics, 144(2):635 - 640.

Ross, S. (1970). Applied Probability Models with Optimality Applications. Holden-Day, San Francisco, CA.

Schmidt, C. and Nahmias, S. (1985). (S-1, S) policies for perishable inventory. Management Science, 31:719-728.

Shang, K. H. and Zhou, S. X. (2010). Optimal and heuristic echelon (r, nQ,T) policies in serial inventory systems with fixed costs. Operations Research, 58(2):414-427.

Springael, J. and Nieuwenhuyse, I. (2005). A lost sales inventory model with a compound poisson demand pattern. Working Paper.

Tekin, E., Gürler, Ü., and Berk, E. (2001). Age-based vs. stock level control policies for perishable inventory system. European Journal of Operational Research, 124:309-329.

van Donselaar, K., Gaur, V., van Woensel, T., Broekmeulen, A., and Fransoo, J. (2010). Ordering behavior in retail stores and implications for automated replenishment. Management Science, 56:766-784.

van Donselaar, K. H. and Broekmeulen, R. A. (2012). Approximations for the relative outdating of perishable products by combining stochastic modeling, simulation and regression modeling. International Journal of Production Economics, 140(2):660 - 669.

Wang, X. and Li, D. (2012). A dynamic product quality evaluation based pricing model for perishable food supply chains. Omega, 40(6):906 - 917 .

Weiss, H. (1980). Optimal ordering policies for continuous review perishable inventory models. Operations Research, 28:365-374.

Williams, C. L. and Patuwo, B. (1999). A perishable inventory model with positive order lead times. European Journal of Operational Rresearch, 116:352-373. 
Williams, C. L. and Patuwo, B. (2004). Analysis of the effect of various unit costs on the optimal incoming quantity in a perishable inventory model. European Journal of Operational Research, 156:140-147.

Xiao, Y. and Chen, J. (2012). Supply chain management of fresh products with producer transportation. Decision Sciences, 43(5):785-815.

Zhou, D., Leung, L., and Pierskalla, W. (2011). Inventory management of platelets in hospitals: Optimal inventory policy for perishable products with regular and optional expedited replenishments. Manufacturing and Service Operations Management, 13(4):420-438. 